\title{
Evaluation of GAD67 immunoreactivity in the region of substantia nigra pars reticulata in resistance to development of convulsive seizure in genetic absence epilepsy rats
}

\author{
Medine Gulcebi,, ${ }^{1}$ Ozlem Akman, ${ }^{1}$ Nihan Carcak, ${ }^{2}$ Tugba Karamahmutoglu, ${ }^{3}$ Filiz Onat $^{1}$ \\ ${ }^{1}$ Department of Pharmacology, Marmara University Faculty of Medicine, Istanbul, Turkey \\ ${ }^{2}$ Department of Physiology, Bilim University Faculty of Medicine, Istanbul, Turkey \\ ${ }^{3}$ Department of Pharmacology, Istanbul University Faculty of Pharmacy, Istanbul, Turkey
}

\begin{abstract}
OBJECTIVE: Nonconvulsive absence epilepsy and convulsive epilepsy seizures are rarely seen in the same patient. It has been demonstrated that there is a resistance to development of convulsive seizures in genetic absence epilepsy models. The present study investigated glutamic acid decarboxylase (GAD) immunoreactivity in the brain region related to the interaction of these two seizure types, namely substantia nigra pars reticulata (SNR) subregions, $\mathrm{SNR}_{\text {anterior }}$ and $\mathrm{SNR}_{\text {posterior" }}$

METHODS: Nonepileptic adult male Wistar rats and Genetic Absence Epilepsy Rats from Strasbourg (GAERS) were used. Experimental groups of Wistar and GAERS were electrically stimulated for kindling model to induce convulsive epileptic seizures. An electrical stimulation cannula was stereotaxically implanted to the basolateral amygdala and recording electrodes were placed on the cortex. Sagittal sections of SNR were used to evaluate immunohistochemical reaction. Sections were incubated with anti-GAD67 antibody. Densitometric analysis of GAD67 immunoreactive neurons was performed using photographs of stained sections. One-way analysis of variance and post hoc Bonferroni test were used for statistical analysis of the data.
\end{abstract}

RESULTS: There was no difference in GAD67 immunoreactivity of SNR subregions of control Wistar and control GAERS. An increase in GAD67 immunoreactivity was detected in $\mathrm{SNR}_{\text {posterior }}$ subregion of stimulated Wistar rats,

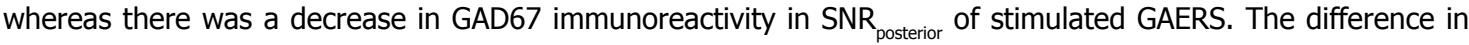
GAD67 immunoreactivity between these two groups was statistically significant.

CONCLUSION: Level of synthetized gamma-aminobutyric acid in $\mathrm{SNR}_{\text {posterior }}$ subregion plays an important role in the interaction of nonconvulsive absence epilepsy seizures and convulsive epilepsy seizures.

Keywords: Epilepsy; GAERS; gamma-aminobutyric acid; immunohistochemistry. 
bsence epilepsy and convulsive epilepsy seizures are rarely seen in the same patient. [1, 2] The mechanisms underlying convulsive seizures are thought to differ from those associated with absence epilepsy seizures. In typical absence epilepsy, inhibitory activities are considered to be dominant factors; however, in focal and generalized seizures, excitatory activities are conceivably predominant factors. Clinical studies and experimental studies have demonstrated concurrent involvement of thalamus and neocortex in formation of spikes and discharges characterized for absence seizures [3, 4]. However in temporal lobe epilepsy, epileptic focus is mostly localized in limbic structures, such as hippocampus, amygdala, or limbic cortex [5]. The genetic models Genetic Absence Epilepsy Rats from Strasbourg (GAERS) and Wistar albino GlaxoRijswijk (WAG-Rij) strain rats meet criteria for absence-type nonconvulsive seizures $[6,7]$. Kindling, generated by regularly repeated stimulations with electrical or chemical stimuli, is frequently used for secondary generalized temporal lobe epilepsy model with convulsive seizures $[8,9]$. Behavioral responses that develop as a result of kindling are evaluated using Racine's scale [10]. There are studies investigating the interaction between convulsive and nonconvulsive seizures which have been done in the amygdala-kindling of GAERS and WAG/ Rij rats. Presence of resistance to generalization of focal limbic seizures and development of convulsive seizures during kindling process in GAERS and WAG/Rij rats was demonstrated [11-18].

Substantia nigra pars reticulata (SNR) is a cerebral region that plays a role in the modulation of both convulsive and nonconvulsive epileptic seizures, and there is an endogenous system associated with SNR which is involved in the control of epileptic seizures [19]. In many studies it has been reported that gamma-aminobutyric acid (GABA)sensitive neurons in SNR mediate convulsive seizures induced in various ways in rats [19-23]. Some studies have shown that through activation or inhibition of SNR by GABAergic agents, direct GABAergic striatonigral pathway can modulate absence epilepsy seizures $[6,19]$. In addition, other studies have demonstrated that SNR contains
2 distinct subregions, $\mathrm{SNR}_{\text {anterior }}$ and $\mathrm{SNR}_{\text {posterior }}$ which may have diverse effects on spread and control of epileptic seizures in adult rats. It has been also reported that just before onset of seizures, there is an increase in deoxyglucose uptake in SN$\mathrm{R}_{\text {posterior }}$ subregion, which may suggest this region as a gateway for spread of seizures [24]. After onset of clonic seizures, an increase in deoxyglucose uptake in the SNR ${ }_{\text {anterior }}$ subregion was detected. Based on various interpretations, it appears that this mechanism may play a role in termination or shortening of seizures [24]. In a recent study of our team, SN$\mathrm{R}_{\text {posterior }}$ was demonstrated to be an important subregion for kindling resistance in GAERS rats [25]. As a result of suppression of $\mathrm{SNR}_{\text {posterior }}$ subregion with lidocaine injections before electrical stimulation, GAERS rats reached stage 5 seizures with repeated kindling stimuli; however, GAERS rats that received lidocaine injection to $\mathrm{SNR}_{\text {anterior }}$ subregion did not experience seizures beyond stage 2, despite recurrent kindling stimuli. Kindling stimuli induced a statistically significant increase in the parvalbumin immunoreactivity of GABAergic interneurons of $\mathrm{SNR}_{\text {posterior }}$ in Wistar rats. Since immunoreactivity of thyrosine hydroxylase in dopaminergic neurons

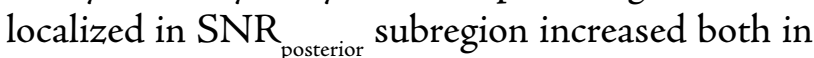
GAERS and Wistar rats, we concluded that thyrosine hydroxylase immunoreactivity is not associated with kindling resistance in GAERS [25]. In our study, it was detected that $\mathrm{SNR}_{\text {posterior }}$ and GABAergic activity of this subregion play a critical role in the resistance to development of convulsive seizures in nonconvulsive genetic epilepsy model. The aim of this study was to analyze the immunoreactivity of glutamic acid decarboxylase (GAD) enzyme, which plays a role in the synthesis of GABA in GABAergic neurons localized in $\mathrm{SNR}_{\text {anterior }}$ and SNRsubregions in control groups and electrically stimulated GAERS and Wistar rats. GAD67 enzyme has a key function in the synthesis of GABA in GABAergic neurons, and levels of GAD enzyme vary in neuropsychiatric diseases. Thus, important information concerning reciprocal interaction between convulsive and nonconvulsive epileptic seizures will be obtained and this will contribute to the clarification of mechanisms underlying epileptic 
seizures and identification of new treatment targets in patients with epilepsy.

\section{MATERIALS AND METHODS}

In the present study, nonepileptic male Wistar rats and GAERS ( 3 to 6 months old and weighing between 250 and 350 g were used. All animals were housed under standard laboratory conditions on a $12 / 12 \mathrm{~h}$ light/dark cycle and were allowed free acess to food and waterApproval of the study was obtained from ethics committee of The Experimental Animal Implementation and Research Centre of Marmara University (March 2, 2011.). Wistar strain rats were procured from experimental research animals unit of Marmara University Faculty of Medicine (DEHAMER), and GAERS were obtained from Marmara University Faculty of Medicine, Department of Medical Pharmacology. Experimental groups were as follows: Electrically stimulated GAERS (stimulated GAERS; $\mathrm{n}=7$ ) and Wistar rats (stimulated Wistar; $n=7$ ), and as control groups, sham-operated GAERS $(n=7)$ and sham-operated Wistar rats $(n=7)$.

\section{Kindling}

All animals were anesthetized with ketamine (100 $\left.\mathrm{mg} / \mathrm{kg}, \mathrm{i} . \mathrm{p}_{+}\right)$, and xylazine hydrochloride $(10 \mathrm{mg} /$ $\mathrm{kg}$, i.p.). Recording electrodes were implanted to cortex and a stimulation electrode into basolateral amygdala (BLA) (anteroposterior: $-2.6 \mathrm{~mm}$, lateral: $4.8 \mathrm{~mm}$, ventral: $-8.5 \mathrm{~mm}$ ) stereotaxically. Animals that received kindling stimulation were stimulated at afterdischarge threshold twice daily for a total of 6 times and reached stage 2 seizure according to $\mathrm{Ra}$ cine's standard 5-stage seizure scale $[10,26]$. Thus, all animals in the stimulation groups were in the transition phase to stage 3 seizures, where convulsive seizures are induced.

\section{Immunohistochemical staining of SNR with GAD67}

Immunohistochemical staining was performed for electrically stimulated GAERS (stimulated GAERS) and Wistar rats (stimulated Wistar), sh- am-operated GAERS, and sham-operated Wistar groups. Stimulated rats were perfused with physiological saline and $4 \%$ neutral buffer formalin solution 1 hour after the sixth stimulation. To achieve standardization, rats in control group that would undergo immunohistochemical staining were perfused on the same day as electrically stimulated rats, and then brains were dissected out. For immunohistochemical staining, $40 \mu \mathrm{m}$-thick, freely floating sagittal sections of SNR were used. For GAD67 staining, sections were first treated with $1 \%$ hydrogen peroxide for 30 minutes. Following washing procedure with phosphate buffer solution (PBS $0.1 \mathrm{M}$ ), sections were blocked in $10 \%$ normal horse serum (in PBS containing $0.1 \%$ TritonX-100, $1 \%$ bovine serum albumin) for 1 hour. Sections were kept at $+4^{\circ} \mathrm{C}$ for 24 hours in PBS solution containing anti-GAD67 antibody at a concentration of $1: 1000$ in combination with $0.3 \%$ TritonX-100 and $1 \%$ bovine serum albumin for primary antibody incubation. Following washing procedure with $0.1 \mathrm{M}$ PBS, sections were incubated for 90 minutes with anti-mouse secondary antibody, then washed again with 0.1 M PBS. Sections were labeled with avidin-biotin complex, and rinsed once more with 0.1 M PBS. NovaRED (Vector Laboratories, Inc., Burlingame, CA, USA) substrate kit was used to visualize staining. Sections were washed with $0.1 \mathrm{M}$ PBS, placed on gelatin-coated slides, and rapid drying was achieved on slide drying hotplate at $37^{\circ} \mathrm{C}$ in period of between 15 and 30 minutes. VectaMount solution (Vector Laboratories, Inc., Burlingame, CA, USA) was added to slides, and they were left to dry at room temperature for at least 1 night. SNR ${ }_{\text {anterior }}$ and $\mathrm{SNR}_{\text {posterior }}$ subregions of stained sections obtained from both groups were photographed and digitally recorded with a digital camera connected to a light microscope. A sample of GAD67 reaction obtained in whole $\mathrm{SN}$, in $\mathrm{SNR}_{\text {anterior }}$ or $\mathrm{SNR}_{\text {posterior }}$ subregions (borders of regions are indicated with arrows) has been shown Figure 1. ImageJ program (US National Institutes of Health, Bethesda, MD, USA) was used for the densitometric analysis of GAD67 immunoreactivity. During analysis of each set, signal intensity of control sham-operated Wistar rat 
was taken as a reference, and results of analyses of other animals in the set were expressed as percent of control Wistar rat.

\section{Histological confirmation}

Histological confirmation was performed by staining 1 set of slices of the brain with thionine. Only rats with properly placed electrodes were included in the study. All data obtained were expressed as means \pm standard error of the mean. For statistical analysis of data, one-way analysis of variance and post hoc Bonferroni test were used. $\mathrm{P}<0.05$ was accepted as the level of significance.

\section{RESULTS}

As a result of comparison between rats with genetic absence epilepsy, namely control GAERS, which demonstrated kindling resistance, and nonepileptic control Wistar rats, a statistically significant difference was not detected for GAD67 immunoreactivity in subregions of $\mathrm{SNR}_{\text {anterior }}$ or $\mathrm{SNR}_{\text {posterior }}$.

GAD67 immunoreactivity in SNR ${ }_{\text {anterior }}$ did not differ between GAERS or Wistar rats following stimulation of BLA (Figure 2). Electrical stimulation had a statistically significant effect on the GAD67 immunoreactivity in $\mathrm{SNR}_{\text {posterior }}$ subregion of stimulated Wistar rats and stimulated GAERS $(p<0.05)$ (Figure 3). GAD67 immunoreactivity increased in $\mathrm{SNR}_{\text {posterior }}$ in stimulated Wistar rats whereas a decrease in GAD67 immunoreactivity was detected in $\mathrm{SNR}_{\text {posterior }}$ of stimulated GAERS group and the difference between these two groups was statistically significant $(\mathrm{p}<0.05)$.

\section{DISCUSSION}

GABA-sensitive neurons found in SNR have an important regulatory role on epilepsy seizures. In addition to its role in the control of convulsive seizures, SNR also assumes role in modulation of nonconvulsive seizures. Many studies have reported on the regulatory role of these neurons in susceptibility of rats against clonic seizures induced with various triggering factors [19-22].

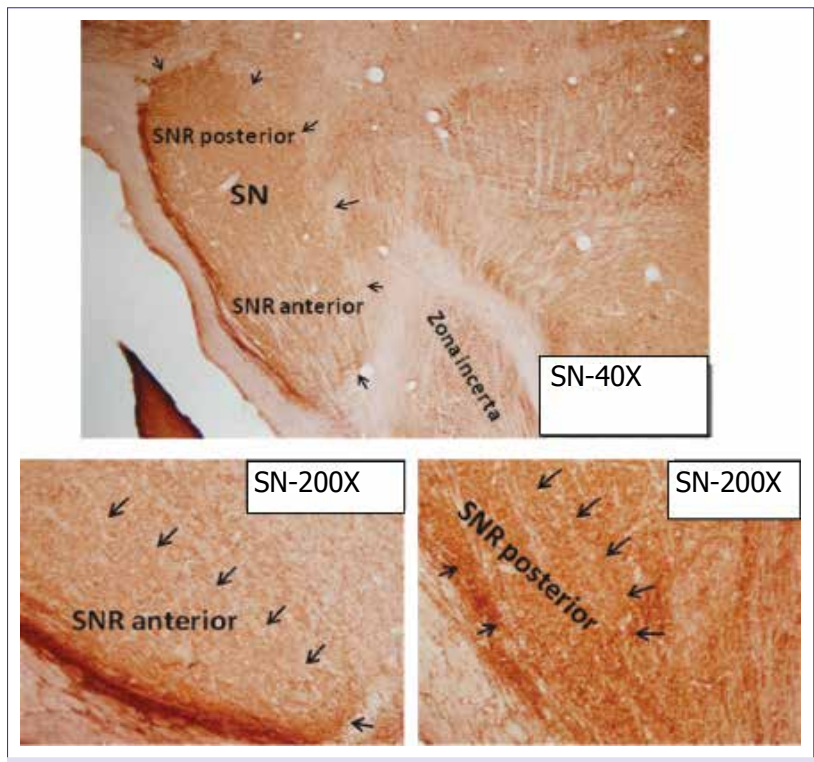

FIGURE 1. Visualization of GAD67 reaction in SN, SNRanterior, and SNR posterior with immunohistochemical staining.

With efferent projections to intrinsic and extrinsic regions, and afferent projections with basal ganglia, SNR plays important roles in both control of epileptic seizures and in the coordination of cognition and motor functions $[22,27,28]$. SNR contains relatively higher amounts of GABA [29]. As a GABAA receptor agonist, muscimol suppresses absence seizures both in genetic and pharmacological absence epilepsy models. However, blockade of GABAA receptors with picrotoxin applied to SNR promoted a development of absence seizures. Furthermore, prevention of absence seizures with application of $\mathrm{N}$-methyl-D-aspartate antagonists to SNR revealed a role of glutamatergic neurotransmission [30]. In adult rats, available evidence has demonstrated the presence of 2 distinct subregions, $\mathrm{SNR}_{\text {anterior }}$ and $\mathrm{SNR}_{\text {posterior }}$ in the SNR, which may have diverse effects on spread and control of seizures. A decrease in activity of GABAergic neurons localized in $\mathrm{SNR}_{\text {anterior }}$ leads to a remission in the severity of seizures. Freichel et al., investigated GABA levels in SNR during kindling, and demonstrated lack of difference in the density of neuronal GABA in SNR measured 6 weeks after stimulation compared with pre-kindling levels. The decrease in GAD and GABA levels detected in previous studies 


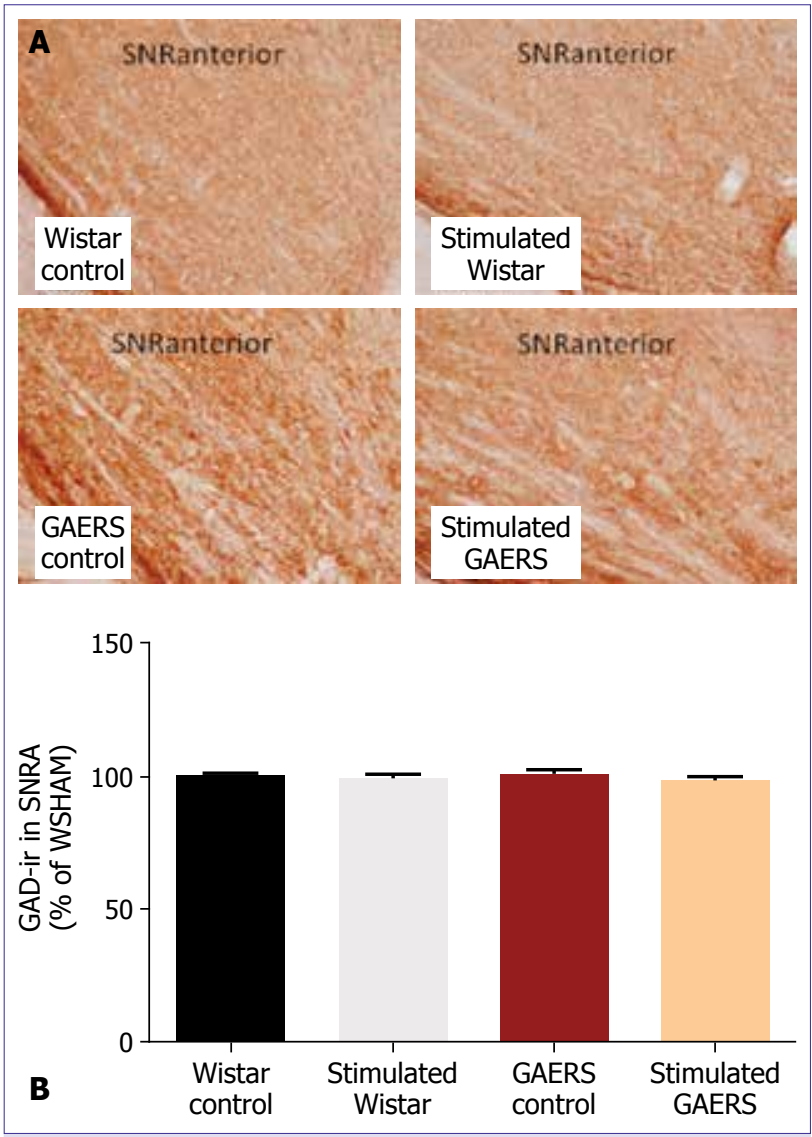

FIGURE 2. GAD67 immunoreactivity in the SNR anterior subregion. (A) Demonstration of GAD67 immunoreactivity in SNR ${ }_{\text {anterior }}$ in the control and stimulated groups. (200x). (B) Comparison of GAD67 immunoreactivity in $\mathrm{SNR}_{\text {anterior }}$ of Wistar rats and GAERS. Mean GAD67 immunoreactivity in $\mathrm{SNR}_{\mathrm{an}}$ terior: Wistar control rats: $100.268 \pm 0.881$; stimulated Wistar rats: $99.408 \pm 1.551$; GAERS control: $100.899 \pm 1.90$; stimulated GAERS: $98.487 \pm 1.230$. Results were expressed as mean \pm standard error. SNRA: SNR $_{\text {anterior }}$

of the same research group were associated with the impact of kindling on GABAergic projections extending from striatum or globus pallidus into SNR, rather than GABAergic neurons in SNR [31].

Based on our results, there was no statistically significant difference in the GAD67 immunoreactivity in SNR ${ }_{\text {anterior }}$ between GAERS and Wistar rats related with the effect of electrical stimulation applied to BLA. Also there was no difference in the GAD67 immunoreactivity in $\mathrm{SNR}_{\text {anterior }}$ between
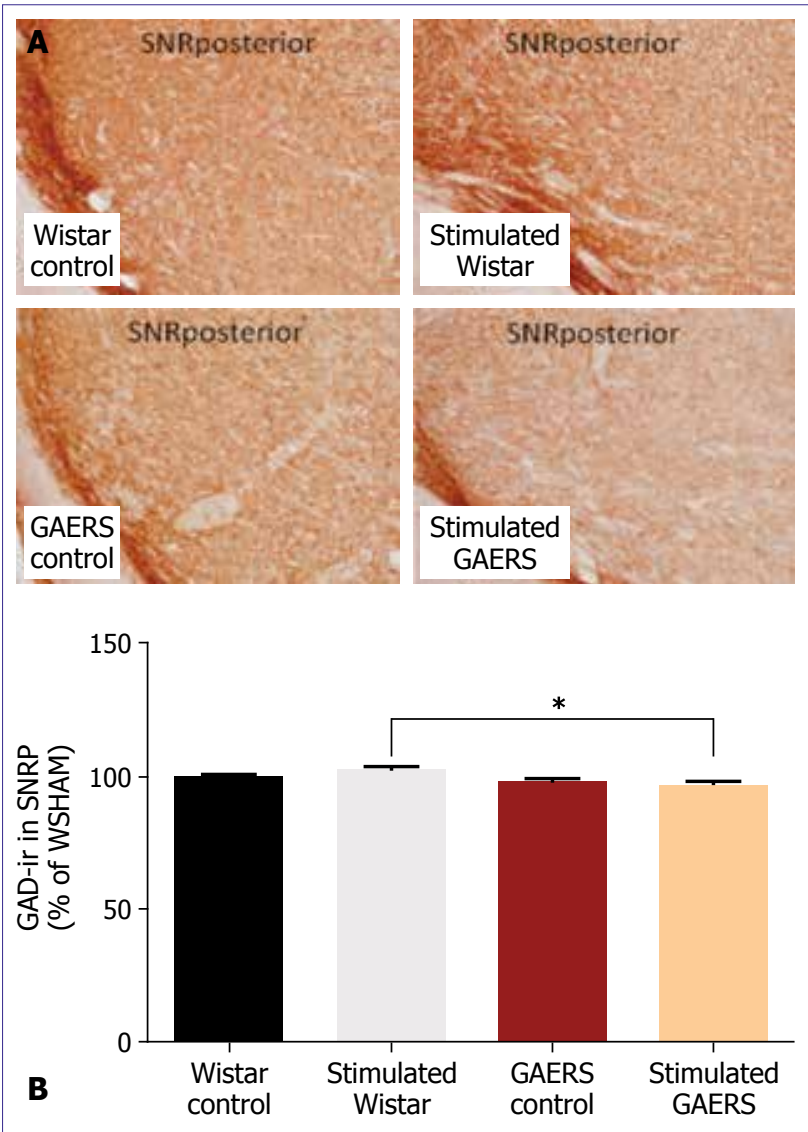

FIGURE 3. GAD67 immunoreactivity in the SNR posterior" (A) Demonstration of GAD67 immunoreactivity in SN$\mathrm{R}_{\text {posterior }}$ in control and stimulated groups. (200x). (B) Comparison of GAD67 immunoreactivity in $\mathrm{SNR}_{\text {posterior }}$ of Wistar and GAERS. Mean GAD67 immunoreactivity in $\mathrm{SNR}_{\text {posterior }}$ : Wistar control rats: $100.162 \pm 1.154$; stimulated Wistar rats: $102.689 \pm 1.321$; GAERS control: 98.319 \pm 0.715 ; stimulated GAERS: $96.979 \pm 1.505$. Results were expressed as mean \pm standard error. Oneway analysis of variance and Bonferroni post hoc test were used for all groups $* ; p<0.05$. SNRP: SNR posterior

control GAERS and Wistar control rats. However, it has not yet been investigated whether analyzed GAD67 immunoreactivity originated from GABAergic neurons of SNR or their projections. In contrast to SNR ${ }_{\text {anterior }}$ GAD67 immunoreactivity in $\mathrm{SNR}_{\text {posterior }}$ differed between stimulated GAERS and stimulated Wistar rats and the difference between these two groups was statistically significant. Although electrical stimulation increased GAD67 immunoreactivity in $\mathrm{SNR}_{\text {posterior }}$ among stimulated 
Wistar ratsa decrease in GAD67 immunoreactivity in the same subregion was determined in stimulated GAERS group. The kindling resistance in GAERS may stem from the difference in GABAergic transmission found in the potentially proconvulsive $\mathrm{SN}$ $\mathrm{R}_{\text {posterior }}$ of the stimulated GAERS. The increase in GAD67 immunoreactivity in SNR posterior $_{\text {pof Wistar }}$ rats following electrical stimulation of BLA might cause an increase in GABAergic transmission and inhibitory effects of SNR on target regions mayresult with easy onset of convulsive seizures. However in stimulated GAERS the decreased GAD67 immunoreactivity in $\mathrm{SNR}_{\text {posterior }}$ might become manifest due to a decrease in GABAergic transmission and a protective effect of SNR on convulsive epileptic seizures. In GAERS, suppressive effect of SNR on epileptic seizures might be more dominant as a result of difference in GAD67 activity in SNR of stimulated GAERS when compared with Wistar rats. In addition, a decrease in GAD67 immunoreactivity in SNR posterior $_{\text {of stimulated GAERS as well }}$ as in GABAergic transmission may lead to abolition of inhibitory effect of SNR in target regions. Consequently, this disinhibition may play role in the prevention of convulsive epileptic seizures in GAERS model due to activation of thalamic nuclei or superior colliculus.

Increased deoxyglucose uptake in $\mathrm{SNR}_{\text {posterior }}$ subregion just before onset of seizure has been reported, and $\mathrm{SNR}$ posterior was considered to be a gateway for the spread of seizure activity [24]. We did not detect any difference between basal values of GABA at nerve terminals in GAERS and Wistar control groups. However, a statistically significant difference in GAD67 immunoreactivity was detect-

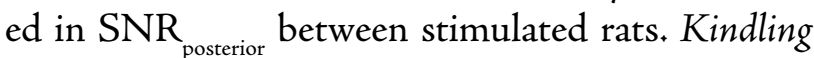
resistance in stimulated GAERS may cause from the difference in GABAergic activity detected in the proconvulsive $\mathrm{SNR}_{\text {posterior }}{ }^{*}$

The results of this study indicated that $\mathrm{SNR}_{\text {pos }}$ has an important role in the interaction of $\mathrm{ab}$ sence epilepsy and temporal lobe epilepsy. In line with the results obtained from our study, analysis of electrophysiological characteristics of SNR and SNR posterior $_{\text {subregions in GAERS and other }}$ models of absence epilepsy during kindling seizure activity, will contribute to highlighting of the clarification of novel underlying mechanisms of epilepsy and identifiying new targets for the effective treatment of seizures.

Conflict of Interest: None declared.

Funding/Sources of funding: This study was supported by the Scientific and Technological Research Council of Turkey (TÜBİTAK; Project no: 111S209).

Authorship contributions: Concept - F.O.; Design - F.O.; Supervision - F.O.; Materials - M.G.; Data collection \&/or processing - M.G.; Analysis and/or interpretation - M.G., Ö.A., N.Ç., T.K., F.O.; Literature search - M.G., Ö.A., N.Ç., T.K.; Writing - M.G., Ö.A., N.C.., T.K.; Critical review - F.O.

\section{REFERENCES}

1. Koutroumanidis M, Hennessy MJ, Elwes RD, Binnie CD, Polkey CE. Coexistence of temporal lobe and idiopathic generalized epilepsies. Neurology 1999;53:490-5. Crossre.

2. Nicholson A, Chadwick DW, Smith DF. The coexistence of idiopatic generalized epilepsy and partial epilepsy. Epilepsia 2004;45:682-5. Crossre

3. Gloor P, Fariello RG. Generalized epilepsy: some of its cellular mechanisms differ from those of focal epilepsy. Trends Neurosci 1988;11:63-8. Crossret

4. Meeren H, van Luijtelaar G, Lopes da Silva F, Coenen A. Evolving concepts on the pathophysiology of absence seizures: the cortical focus theory. Arch Neurol 2005;62:371-6. Crossret

5. Engel JrJ. Introduction to temporal lobe epilepsy. Epilepsy Research 1996;26:141-50. Crossre

6. Depaulis A, Snead OC 3rd, Marescaux C, Vergnes M. Suppressive effects of intranigral injection of muscimol in three models of generalized non-convulsive epilepsy induced by chemical agents. Brain Res 1989;498:64-72. Crossre

7. van Luijtelaar EL, Coenen AM. Two types of electrocortical paroxysms in an inbred strain of rats. Neuroscience Letters 1986;70:393-7. Crossret

8. Bertram EH. Temporal lobe epilepsy: Where do the seizures really begin? Epilepsy \& Behav 2009;14:32-7. Crossre.

9. Mcintyre DC, Poulter MO, Gilby K. Kindling: some old and some new. Epilepsy Research 2002;50:79-92.

10. Racine RJ. Modification of seizure activity by electrical stimulation. II. Motor seizure. Electroencephalography and Clinical Neurophysiology 1972;32:281-94. Crossre

11. Eskazan E, Onat FY, Aker R, Öner G. Resistance to propagation of amygdaloid kindling seizures in rats with genetic absence epilepsy. Epilepsia 2002;43:1115-1. Crossre-

12. Onat FY, Eşkazan E, Aker R. Experimental absence versus amygdaloid kindling. Advances in Behavioral Biology, Kindling 6. Editörler: M. Corcoran, SL Moshe, Springer, 2005. p. 37-48. 
13. Aker RG, Yananli HR, Gurbanova AA, Ergun AO, Ates N, van Luijtelaar G, et al. Amygdala kindling in the WAG/Rij rat model of absence epilepsy. Epilepsia 2006;47:33-40. Crossre

14. Onat FY, Aker RG, Gurbanova AA, Ateş N, van Luijtelaar G. The effect of generalized absence seizures on the progression of kindling in the rat. Epilepsia 2007;48:150-6. Crossref

15. Çarçak N, Ferrandon A, Koning E, Aker GA, Özdemir O, Onat FY, et al. Effect of stage 2 kindling on local cerebral blood flow rates in rats with genetic absence epilepsy. Epilepsia 2009;50:3343. Crossret

16. Onat FY, Eşkazan E, Aker R. Interactions between CorticoThalamo-Cortical and Limbic Seizures. Philip A. Schwartzkroin, editor Encyclopedia of Basic Epilepsy Research. Oxford: Academic Press 2009;2:825-30. Crossret

17. Akman O, Karson A, Aker RG, Ates N, Onat FY. Hippocampal kindling in rats with absence epilepsy resembles amygdaloid kindling. Epilepsy Research 2008;81:211-9. Crossret

18. Akman O, Karson A, Aker RG, Ates N, Onat FY. Perirhinal cortical kindling in rats with genetic absence epilepsy. Neuroscience Letters, 2010;479:74-8. Crossre

19. Depaulis A, Vergnes M, Marescaux C. Endogenous control of epilepsy: the nigral inhibitory system. Progress in neurobiology 1994;42:33,52.

20. Bloms-Funke P, Löscher W. The anticonvulsant gabapentin decreases firing rates of substantia nigra pars reticulata neurons. Eur J Pharmacol 1996;316:211-8. Crossre

21. Gale K. Mechanisms of seizure control mediated by gammaaminobutyric acid: role of the substantia nigra. Federation proceedings 1985;44:2414-24.

22. Mcnamara JO, Galloway MT, Rigsbee LC, Shin C. Evidence im- plicating substantia nigra in regulation of kindled seizure threshold. Journal of Neuroscience 1984;4:2410-7.

23. Moshe SL, Sperber EF. Substantia nigra-mediated control of generalized seizures, Generalized epilepsy: cellular, molecular and pharmacological approaches. Ed: Gloor G, Kostopoulos R, Naquet M, Avoli P. Birkhauser Inc, Boston 1990;355-67.

24. Veliskova J, Miller AM, Nunes ML, Brown LL. Regional neural activity within the substantia nigra during peri-ictal flurothyl generalized seizure stages. Neurobiology of Disease 2005;20:752-9. Crossret

25. Akman O, Gulcebi MI, Carcak N, Ketenci Ozatman S, Eryigit T, Moshé SL, et al. The role of the substantia nigra pars reticulata in kindling resistance in rats with genetic absence epilepsy. Epilepsia 2015;56:1793-802. Crossre

26. Racine RJ. Modification of seizure activity by electrical stimulation. I. After-discharge threshold. Electroencephalography and Clinical Neurophysiology 1972;32:269-79. Crossre,

27. Iadarola MJ, Gale K. Substantia nigra: site of anticonvulsant activity mediated by $\gamma$ aminobutyric acid. Science 1982;218:1237-40.

28. Veliskova J, Moshé SL. Update on the role of substantia nigra pars reticulata in the regulation of seizures. Epilepsy Currents 2006;6:83-7. Crossre

29. Bolam JP, Hanley JJ, Booth PA, Bevan MD. Synaptic organisation of the basal ganglia. Journal of Anatomy 2000;196:527-42.

30. Deransart C, Marescaux C, Depaulis A. Involvement of nigral glutamatergic inputs in the control of seizures in a genetic model of absence epilepsy in the rat. Neuroscience 1996;71:721-8.

31. Freichel C, Ebert U, Potschka H, Löscher W. Amygdala-kindling does not induce a persistent loss of GABA neurons in the substantia nigra pars reticulata of rats. Brain Res 2004;29:1025:203-9. 